\title{
Adverse events during oral colchicine use: a systematic review and meta-analysis of randomised controlled trials
}

\author{
Sarah Stewart ${ }^{1}$, Kevin Chih Kai Yang ${ }^{2}$, Kate Atkins ${ }^{1}$, Nicola Dalbeth ${ }^{1}$ and Philip C. Robinson ${ }^{2,3^{*}}$ (D)
}

\begin{abstract}
Background: Colchicine is a widely used drug to treat inflammatory diseases. Due to its long historical use in medicine, controlled clinical trials have been small and there remains some caution with the use of this drug in patients with co-morbidities. The aim of the study is to systematically examine the side effect profile of colchicine in controlled clinical trials across all published indications.

Methods: A systematic review was conducted in accordance with PRISMA methodology. The Cochrane Library, MEDLINE and EMBASE were searched for double-blind controlled trials of oral colchicine in adult patients that reported adverse event data. Meta-analyses were used to determine the relative risk (RR) of adverse events in colchicine users compared to comparator groups.

Results: A total of 4915 studies were initially identified and after exclusions, 35 randomised controlled trials with placebo $(n=30)$ or active comparators $(n=5)$ were included. The most common diseases studied were gout, liver cirrhosis and pericarditis. There were a total of 8659 pooled participants, 4225 participants were randomised to receive colchicine, 3956 to placebo and 411 to an active comparator. Diarrhoea was reported in $17.9 \%$ of colchicine users versus $13.1 \%$ in comparator groups (RR 2.4, 95\% confidence interval (Cl) 1.6, 3.7). Any gastrointestinal event was reported in $17.6 \%$ of colchicine users and $13.1 \%$ of comparators (RR 1.7, 95\% Cl 1.3,2.3). Adverse liver events were reported in $1.9 \%$ of colchicine users versus $1.1 \%$ in the comparator groups (RR 1.6, $95 \% \mathrm{Cl} 0.9,3.0$ ). Muscle events were reported in $4.2 \%$ of colchicine users and $3.3 \%$ in the comparator groups (RR 1.3,95\% Cl 0.8, 1.9). Haematology events were reported in $0.6 \%$ of colchicine users and $0.4 \%$ of comparator groups (RR $1.34(0.64,2.82)$. No study reported neuropathy events. Other sensory events were reported in $1.1 \%$ of colchicine users and $1.5 \%$ of comparator groups (RR 1.4, 95\% Cl 0.3,6.7). Infectious events were reported in $0.4 \%$ of colchicine users and $2.1 \%$ of comparator groups (RR 1.0, 95\% Cl 0.7, 1.5). No study reported death as an adverse event.
\end{abstract}

Conclusion: Colchicine increases the rate of diarrhoea and gastrointestinal adverse events but does not increase the rate of liver, sensory, muscle, infectious or haematology adverse events or death.

Keywords: Colchicine, Gout, Diarrhoea, Nausea

\footnotetext{
*Correspondence: philip.robinson@uq.edu.au

${ }^{2}$ School of Clinical Medicine, Faculty of Medicine, University of Queensland,

Brisbane, Australia

${ }^{3}$ Department of Rheumatology, Royal Brisbane Hospital, Bowen Bridge Road,

Herston, QLD 4006, Australia

Full list of author information is available at the end of the article
}

(c) The Author(s). 2020 Open Access This article is distributed under the terms of the Creative Commons Attribution 4.0 International License (http://creativecommons.org/licenses/by/4.0/), which permits unrestricted use, distribution, and reproduction in any medium, provided you give appropriate credit to the original author(s) and the source, provide a link to the Creative Commons license, and indicate if changes were made. The Creative Commons Public Domain Dedication waiver (http://creativecommons.org/publicdomain/zero/1.0/) applies to the data made available in this article, unless otherwise stated. 


\section{Introduction}

Colchicine is an anti-inflammatory agent which is widely used for the treatment of gout and also used extensively for familial Mediterranean fever, Behcet's disease and pericarditis. Its use in the management of gout has increased due to the widespread recommendation that it be used as a gout flare prophylaxis when urate-lowering therapy is initiated [1]. It is used continuously for long periods of time in individuals with familial Mediterranean fever and Behcet's disease. However, due to its long historical use in medicine, it has not been subjected to the same registration trials that contemporary medicines require. There remains uncertainty regarding its use in certain risk groups including those with kidney and liver impairment, at higher doses, and with CYP3A4 inhibitors [2]. It has previously been used in an intravenous preparation, but this is no longer used due to the adverse safety profile of this administration method [3].

Although the adverse event profile of colchicine has been reported in various individual clinical trials and for single indications like pericarditis [4], it has not been studied systematically to our knowledge. The aim of this study was to examine the adverse events of colchicine reported in randomised controlled trials using a systemic review and meta-analysis methodology.

\section{Methods}

This study was conducted according to a pre-defined protocol using the Preferred Reporting Items for Systematic Reviews and Meta-Analyses (PRISMA) statement [5].

\section{Search strategy}

Electronic databases (Cochrane Library, MEDLINE and EMBASE) were searched from inception to November 2019 using the following key words in the search term: colchicine AND ((randomised controlled trial [pt] OR controlled clinical trial [pt] OR randomised [tiab] OR placebo [tiab] OR drug therapy [sh] OR randomly [tiab] OR trial [tiab] OR groups [tiab]) NOT (animals [mh] NOT humans $[\mathrm{mh}])$ ). Bibliographical references from individual included studies and review articles were also hand-searched to identify additional relevant papers. All studies generated from the search were exported to RefWorks and screened to remove duplicates. Title and abstract screening, followed by full-text screening, was undertaken by a single reviewer (KA). Randomised controlled trials were included if they compared the effects of colchicine in patients, administered for any indication, to placebo or active comparators. Trials were included if they: had a double-blinded design, included oral colchicine in at least one of the treatment arms; involved adult participants; were published in the English language; and reported adverse event data in both the colchicine and comparator group(s) in relation to the number of participants with adverse events per group. Studies which reported the number of participants with adverse events resulting in study withdrawals and did not report the number of adverse events which occurred in the participants who remained in the study, were excluded. There was no publication date restriction. If multiple reports described the same trial, the most recent full-text publication was selected for inclusion.

\section{Quality assessment}

Quality of all included studies was assessed independently by two reviewers (SS, KY) using the 6-item modified-Jadad scale which assesses reported randomisation, blinding, withdrawals, dropouts, inclusion/exclusion criteria, adverse events and the statistical analysis [6]. The scale has a maximum score of 8 points, with low quality studies yielding scores of 0 to 3 and highquality studies yielding a score of 4 to 8 . Any disagreements in the quality assessment were resolved by discussion of the two reviewers. If necessary, a third reviewer (ND) was involved to reach consensus.

\section{Data extraction}

Two reviewers (SS, KY) independently extracted data from the full-text studies using a Microsoft Excel extraction form. Any disagreements were resolved by consensus with a third reviewer (ND). Data extraction included publication details (author, year of publication, country of first author), disease state, participant characteristics (sample size, ethnicity, mean (SD) age and $\mathrm{n} \%$ of male participants) and details of the trial (study design, length of follow up, primary outcome, interventions and dosages, intervention length). Extraction of data related to adverse events included the total number of participants with any adverse event per group and the total number of participants with each individual reported adverse event. Data for the number of deaths were extracted only if death was related to an adverse event (rather than worsening of disease).

\section{Data-analyses}

For the purpose of data analysis, adverse events were grouped under eight pre-defined categories: diarrhoea, gastrointestinal events (including diarrhoea), liver events, haematology events, muscle-related events, sensoryrelated events (including neuropathy), infectious events, death and any adverse event. In situations when studies reported the number of participants with $\geq 2$ individual adverse events which both came under the same category (i.e. "nausea" and "vomiting" which both come under the gastrointestinal event category), data from the adverse event with the highest number of participants was used for that category. Only studies which reported 
diarrhoea as a separate event were included in the diarrhoea category. Studies which reported diarrhoea as part of a combined event (i.e. 'diarrhoea or nausea') were included under the 'gastrointestinal events' category.

Meta-analyses were undertaken to determine the relative risk of adverse events in the colchicine group compared to the comparator groups (pooled comparators, placebo and active-comparators). Relative risk was calculated based on the number of participants with adverse events. Random effects models were used for all $I^{2}$ values $>0 \%$. As caution is recommended when colchicine is used continuously in those with liver impairment, a sensitivity analysis was undertaken excluding the studies involving participants with cirrhosis or sclerosing cholangitis [6-11]. For the 'any adverse events' category, meta-analyses were also used to determine the effects of disease indication (liver diseases, gout, Behcet's and related conditions, pericarditis and related conditions and other), duration of exposure to the intervention (with subgroups defined as $\leq 2$ weeks, 1 to 2 months, 3 to 5 months, 6 to 12 months and $\geq 24$ months), the average daily dose of colchicine (with subgroups defined as $<1$ $\mathrm{mg}, 1 \mathrm{mg},>1<2 \mathrm{mg}, \geq 2 \mathrm{mg}$ ) and the cumulative daily dose of colchicine (with subgroups defined as $<50 \mathrm{mg}$, 50 to $<100 \mathrm{mg}, \geq 100 \mathrm{mg}$ to $300 \mathrm{mg},>600 \mathrm{mg}$ ). In papers which used different colchicine doses based on participant weight categories, the highest daily colchicine dose was used to determine that study's subgroup. Subgroup comparisons were made using the Phet statistic (the $P$ value derived from the chi-square test of heterogeneity for subgroup differences).

All meta-analyses were undertaken in Review Manager 5.3 with an alpha level of 0.05 . Only studies specifically reporting an adverse event as being present or absent were included in the meta-analyses. However, as this may over- or under-estimate the true occurrence of adverse events, the proportion of participants with specific adverse events was also computed in relation to the total number of participants included in all studies (i.e. if not reported, ' 0 ' events were considered to have occurred). These data were used for descriptive purposes only and not meta-analysed.

\section{Results}

\section{Study characteristics}

A total of 4915 studies were identified through the search following the deletion of duplicates (Fig. 1). After title and abstract screening, 70 full-text articles were assessed for eligibility. After the exclusion of 35 studies (reasons for exclusion are presented in Fig. 1), a total of 35 randomised-controlled double-blind studies were included in this review. The majority of studies were placebo-controlled $(n=30,83 \%)$ and $5(17 \%)$ studies were active-comparator controlled. The majority of studies were parallel-group designs and 4 studies [12$15]$ were cross-over designs.

\section{Quality assessment}

The results from the quality assessment are presented in Supplementary Figure 1. Overall, the modified-Jadad score indicated high quality (total score 4 to 8 ) for all studies. An appropriate method of randomisation and blinding was reported in 24 (69\%) and 28 (80\%) studies, respectively. Thirty (86\%) studies provided an adequate description of withdrawals and dropouts and 33 (94\%) provided a participant inclusion and exclusion criteria. The method used to assess adverse events was described by $22(63 \%)$ studies and methods of statistical analysis by $32(91 \%)$.

\section{Participant characteristics}

Characteristics of participants in the included studies are shown in Table 1. A number of disease states were studied including cirrhosis ( $n=5$ studies) [6-10], pericarditis ( $n=4$ studies) [26, 27, 29,31], gout ( $n=5$ studies) [15, $18,34,38,39]$, knee osteoarthritis ( $n=3$ studies) [16, 20, $30]$, Behcet's syndrome ( $n=3$ studies) [21, 32, 40], psoriatic arthritis ( $n=2$ studies) [13,36], post-pericardiotomy syndrome ( $n=2$ studies) [25, 28], chronic obstructive pulmonary disorder ( $n=1$ study) [19], bare-metal stent restenosis ( $n=1$ study) [22], metabolic syndrome $(n=1$ study) [23], lung resection surgery ( $n=1$ study) [17], myocardial infarction ( $\mathrm{n}=1$ study) [37], familial Mediterranean fever ( $n=1$ study) [12], asthma ( $n=1$ study) [24], primary sclerosing cholangitis $(n=1$ study) [11], aphthous stomatitis ( $n=1$ study) [33], allergic rhinitis ( $n=1$ study) [14] and low back pain ( $n=1$ study) [35]. Sample sizes ranged from 11 to 4745 , with a pooled sample of 8659 adult participants. Mean age ranged from 27.0 to 69.1 years with most participants being male $(73 \%)$. The inclusion and exclusion criteria reported by the included studies are shown in Supplementary Table 1.

\section{Intervention characteristics}

Of the 8659 pooled participants, 4225 participants were randomised to receive colchicine, 3956 to the placebo and 411 to an active comparator. The remaining 67 participants were included in cross-over trials and received both colchicine and placebo treatments over the duration of the study [12-15].

The length of treatment varied across studies (Table $1)$ ). The majority of studies administered treatment for $\geq 1$ to $\leq 6$ months ( $n=16$ studies) $[13,15,16,20,21$, $23-25,27,28,30-33,35,36],>6$ to $\leq 12$ months $(n=6$ studies) $[12,18,22,26,29,34]$ or $>12$ to $\leq 48$ months ( $n=6$ studies) $[6,8,9,11,37,40]$. Four studies administered treatment for one to 2 weeks $[14,17,19,39]$ and 


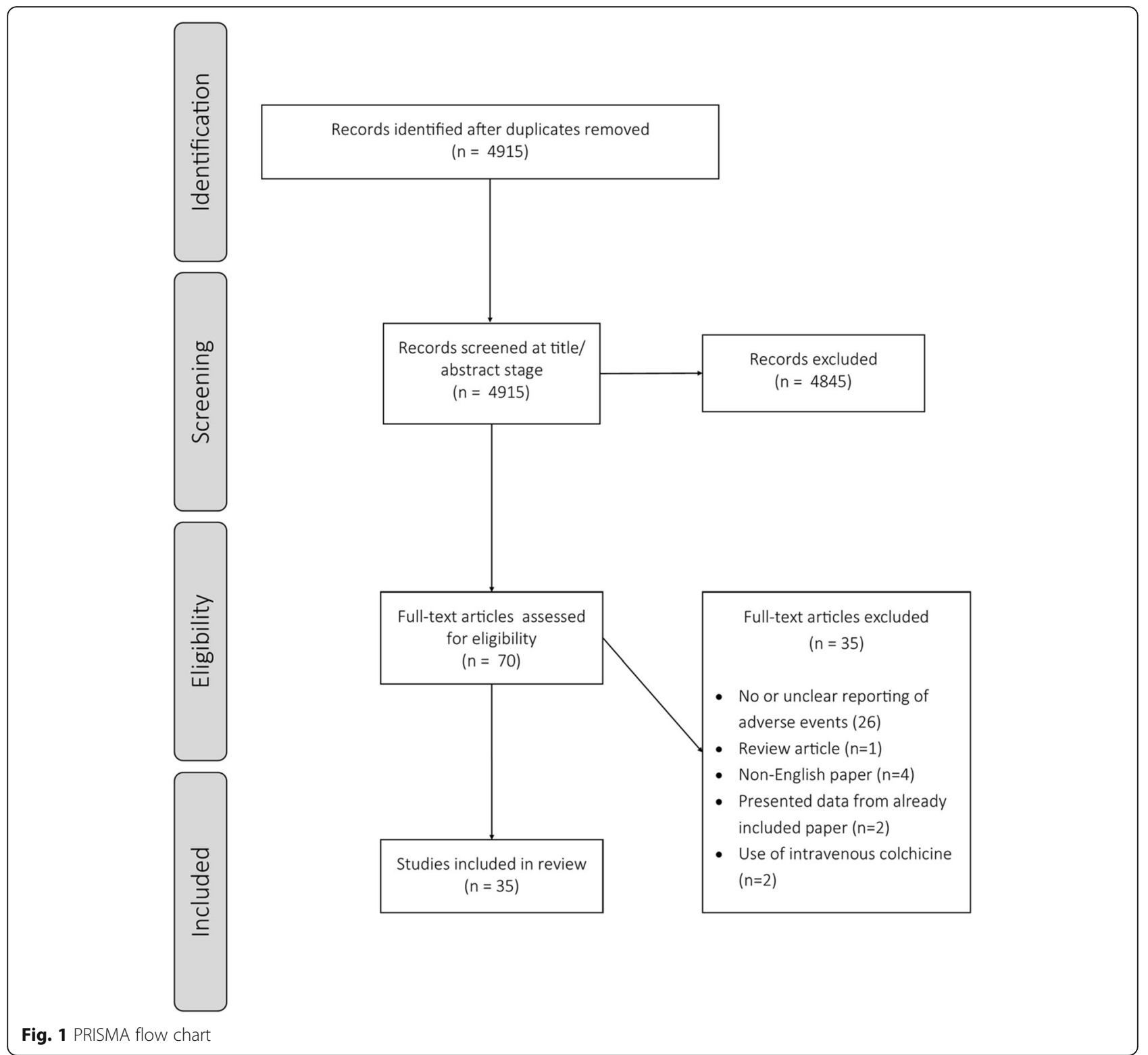

participants in one study received treatment for one to $6 \mathrm{~h}$ [38]. Participants in two studies received treatment for $\geq 10$ years $[7,10]$. The mean daily dose of colchicine ranged from $0.5 \mathrm{mg}$ to $4.8 \mathrm{mg}$. One study [38] reported the difference in the number of participants with adverse events based on whether they received low dose colchicine (total dose $1.8 \mathrm{mg}$ ) or high dose colchicine (total dose $4.8 \mathrm{mg}$ ).

\section{Adverse events}

Methods used in the included studies to assess adverse events are described in Supplementary Table 1. Assessment methods included self-reporting of symptoms by patients, questioning of adverse events by investigators during study visits and undertaking blood tests and laboratory analyses.

\section{Any adverse event}

The number of participants with any adverse event was reported by 27 papers (Supplementary Table 2). From this data, $21.1 \%$ (95\% confidence interval (CI) 19.9, 22.4) of participants using colchicine reported any adverse event compared to $18.9 \%$ (95\% CI 17.7 , 20.1) of participants in comparator groups. A metaanalysis showed the overall estimated risk ratio (RR) ( $95 \%$ CI) of any adverse event in colchicine users compared with pooled comparator groups was 1.46 (1.20, 1.77), $P<0.001$ (Fig. 2, Table 2). The difference in RR of any adverse event in colchicine users was not 


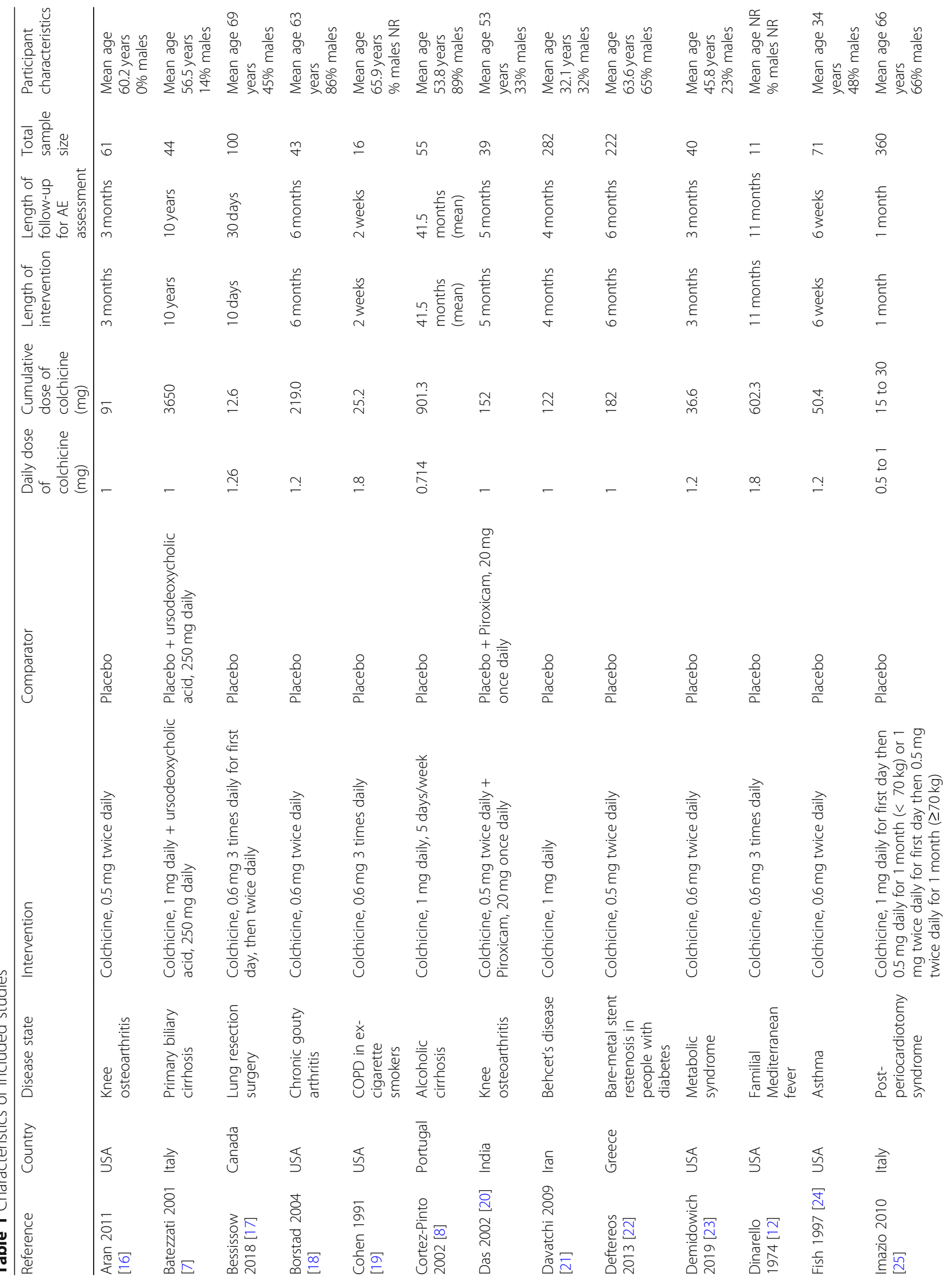




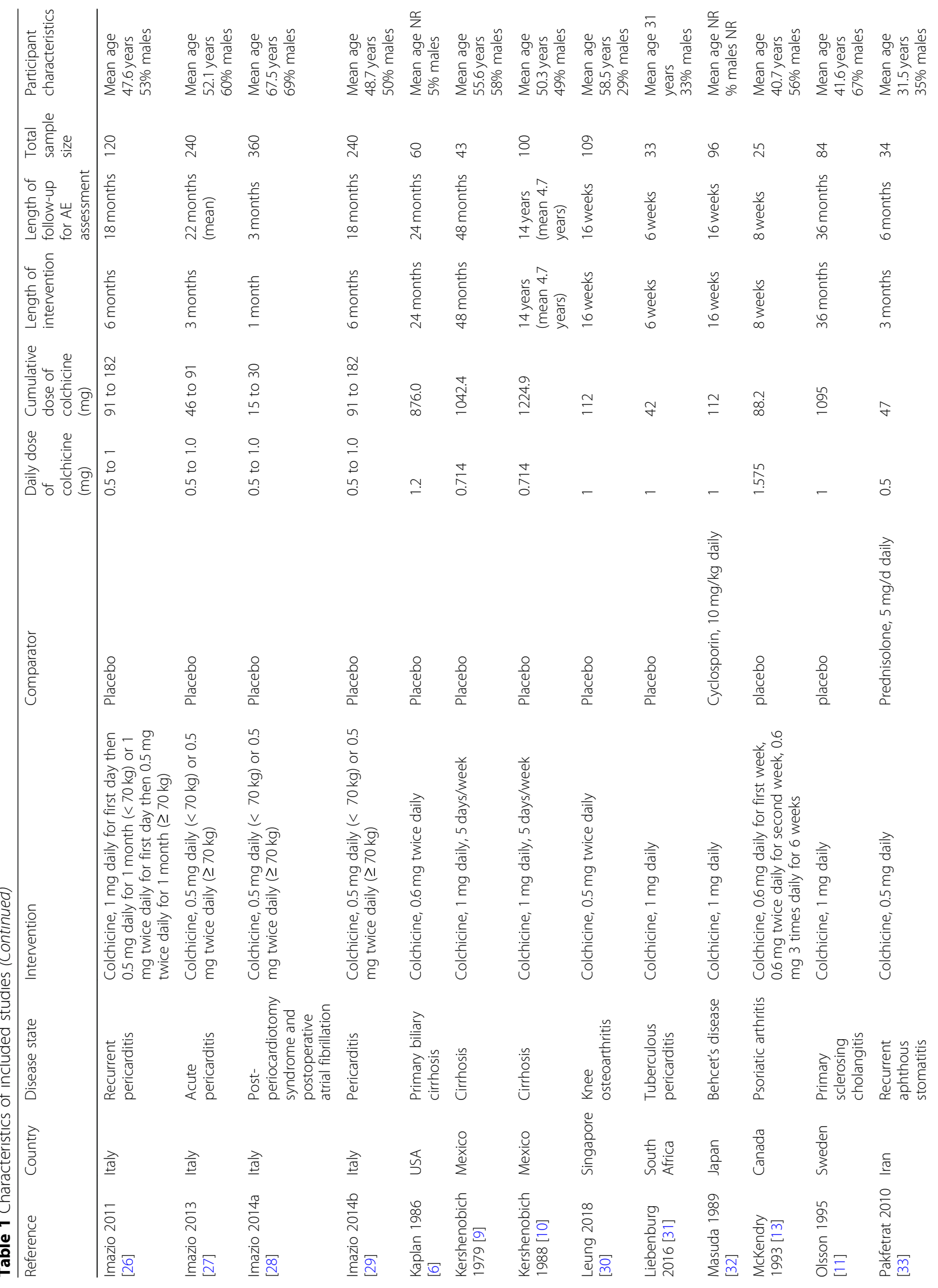




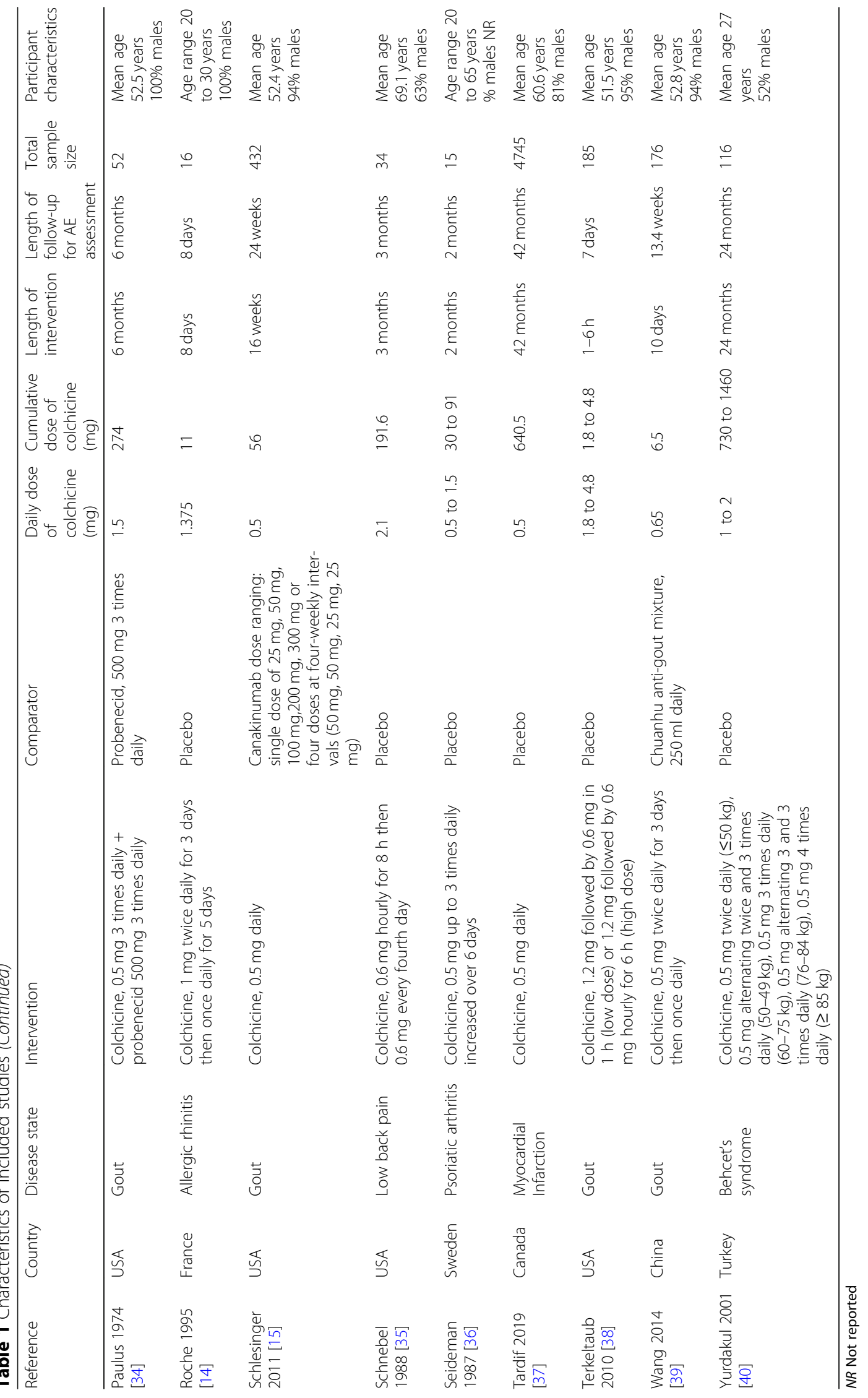




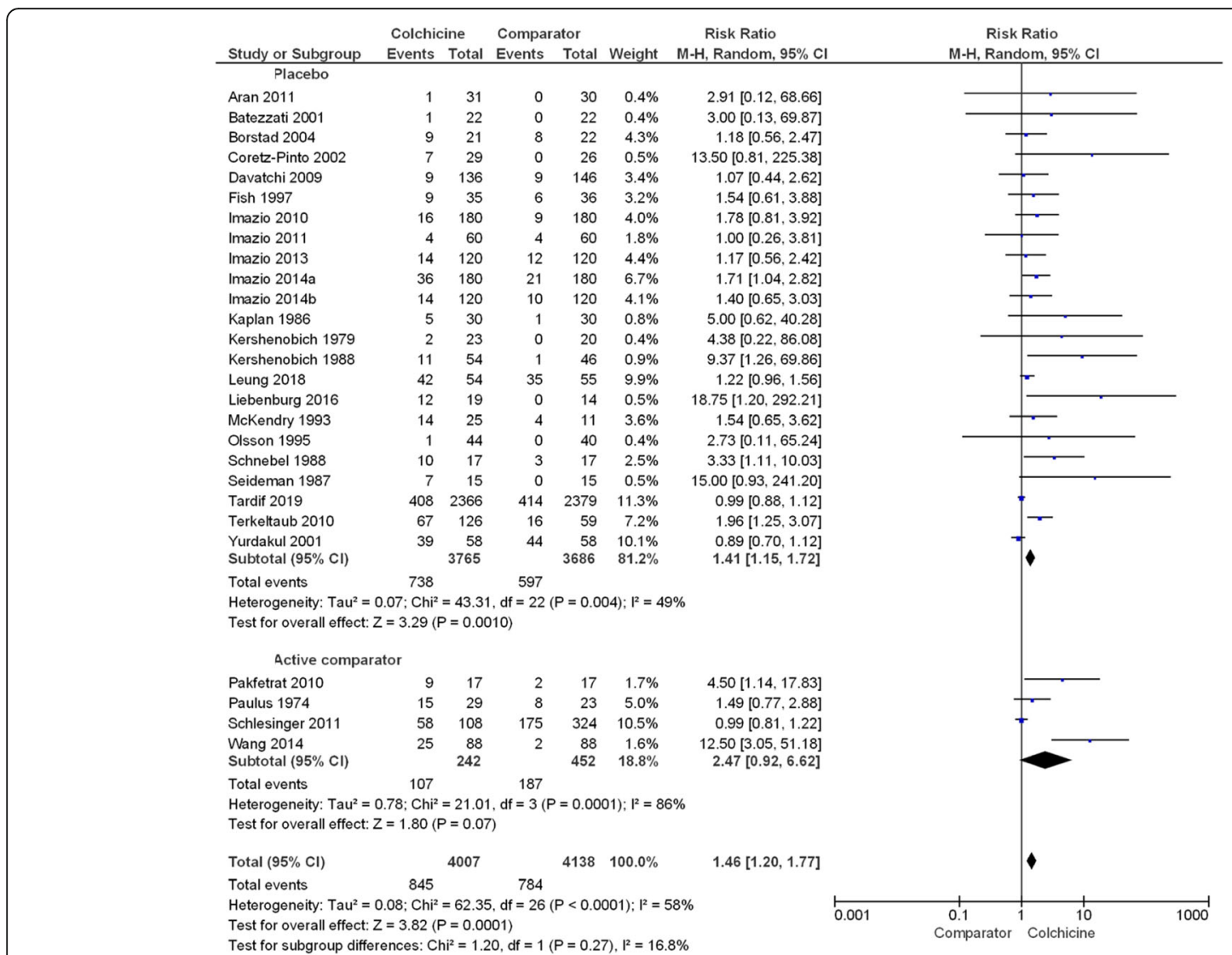

Fig. 2 Forest plot showing estimated relative risk of any adverse event during colchicine use compared to placebo and active comparator groups

significantly different between placebo and active comparator groups $(P=0.27)$. After the exclusion of six studies involving participants with liver disease, the RR (95\% CI) of any adverse event in colchicine users vs. comparator groups was similar at $1.37(1.14,1.65), P<$ 0.001 (Supplementary Table 3).
Although the sub-group meta-analyses showed a higher relative risk for any adverse event in colchicine users with liver diseases (RR 5.92 (95\% CI 2.08, 16.82)), there was no overall significant difference in the relative risk of adverse events between different disease indications $(P=0.11)$ (Fig. 3). Furthermore,

Table 2 Meta-analysis results showing pooled risk ratio of adverse events between colchicine and pooled comparator groups

\begin{tabular}{|c|c|c|c|c|c|c|}
\hline & \multirow{2}{*}{$\begin{array}{l}N . \\
\text { studies }\end{array}$} & \multicolumn{2}{|l|}{ n/N, \% (95\% Cl) participants } & \multirow{2}{*}{$\begin{array}{l}\text { Pooled risk } \\
\text { ratio }(95 \% \mathrm{Cl})\end{array}$} & \multirow[t]{2}{*}{$P^{2}$ ( $P$ value $)$} & \multirow{2}{*}{$\begin{array}{l}\text { Overall effect, } \\
Z{\text { ( } P \text { value })^{\mathrm{a}}}^{\text {a }}\end{array}$} \\
\hline & & Colchicine & Comparator & & & \\
\hline Any event & 27 & $845 / 4007,21.1 \%(19.9,22.4)$ & $784 / 4152,18.9 \%(17.7,20.1)$ & $1.46(1.20,1.77)$ & $58 \%(<\mathbf{0 . 0 0 1})$ & $3.82(<\mathbf{0 . 0 0 1 )}$ \\
\hline Diarrhoea & 19 & $420 / 3212,17.9 \%(16.8,19.1)$ & $262 / 3142,13.1 \%(11.9,14.3)$ & $2.44(1.62,3.69)$ & $58 \%(<\mathbf{0 . 0 0 1})$ & $4.24(<\mathbf{0 . 0 0 1 )}$ \\
\hline Gastrointestinal ${ }^{b}$ & 29 & $729 / 4131,17.6 \%(16.5,18.8)$ & $552 / 4213,13.1 \%(12.1,14.2)$ & $1.74(1.32,2.30)$ & $53 \%(<\mathbf{0 . 0 0 1})$ & $3.94(<0.001)$ \\
\hline Liver & 13 & $22 / 1150,1.9 \%(1.2,2.8)$ & $15 / 1362,1.1 \%(0.6,1.8)$ & $1.61(0.86,3.02)$ & $0 \%(0.48)$ & $1.50(0.13)$ \\
\hline Muscle ${ }^{c}$ & 9 & $37 / 872,4.2 \%(3.0,5.7)$ & $29 / 869,3.3 \%(2.3,4.7)$ & $1.25(0.80,1.93)$ & $0 \%(0.69)$ & $0.98(0.33)$ \\
\hline Haematology & 8 & $16 / 2878,0.6 \%(0.3,0.9)$ & $12 / 28930.4 \%(0.2,0.7)$ & $1.34(0.64,2.82)$ & $0 \%(0.69)$ & $0.77(0.44)$ \\
\hline Sensory $^{d}$ & 2 & $3 / 201,1.5 \%(0.4,4.0)$ & $2 / 190,1.1 \%(0.2,3.4)$ & $1.35(0.27,6.74)$ & $0 \%(0.58)$ & $0.37(0.71)$ \\
\hline Infectious & 7 & $105 / 2763,3.8 \%(3.1,4.6)$ & $131 / 2997,4.4 \%(3.7,5.1)$ & $1.03(0.70,1.51)$ & $46 \%(0.09)$ & $0.13(0.90)$ \\
\hline
\end{tabular}

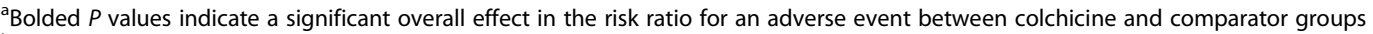

${ }^{\mathrm{b}}$ The gastrointestinal category includes diarrhoea

'The muscle category includes myalgia, muscle cramps, myotoxicity, muscle weakness and elevated CPK. No rhabdomyolysis was assessed or reported by any study

${ }^{\mathrm{d}}$ The sensory category includes dysthesia and paresthesia. No neuropathy was assessed or reported by any study 


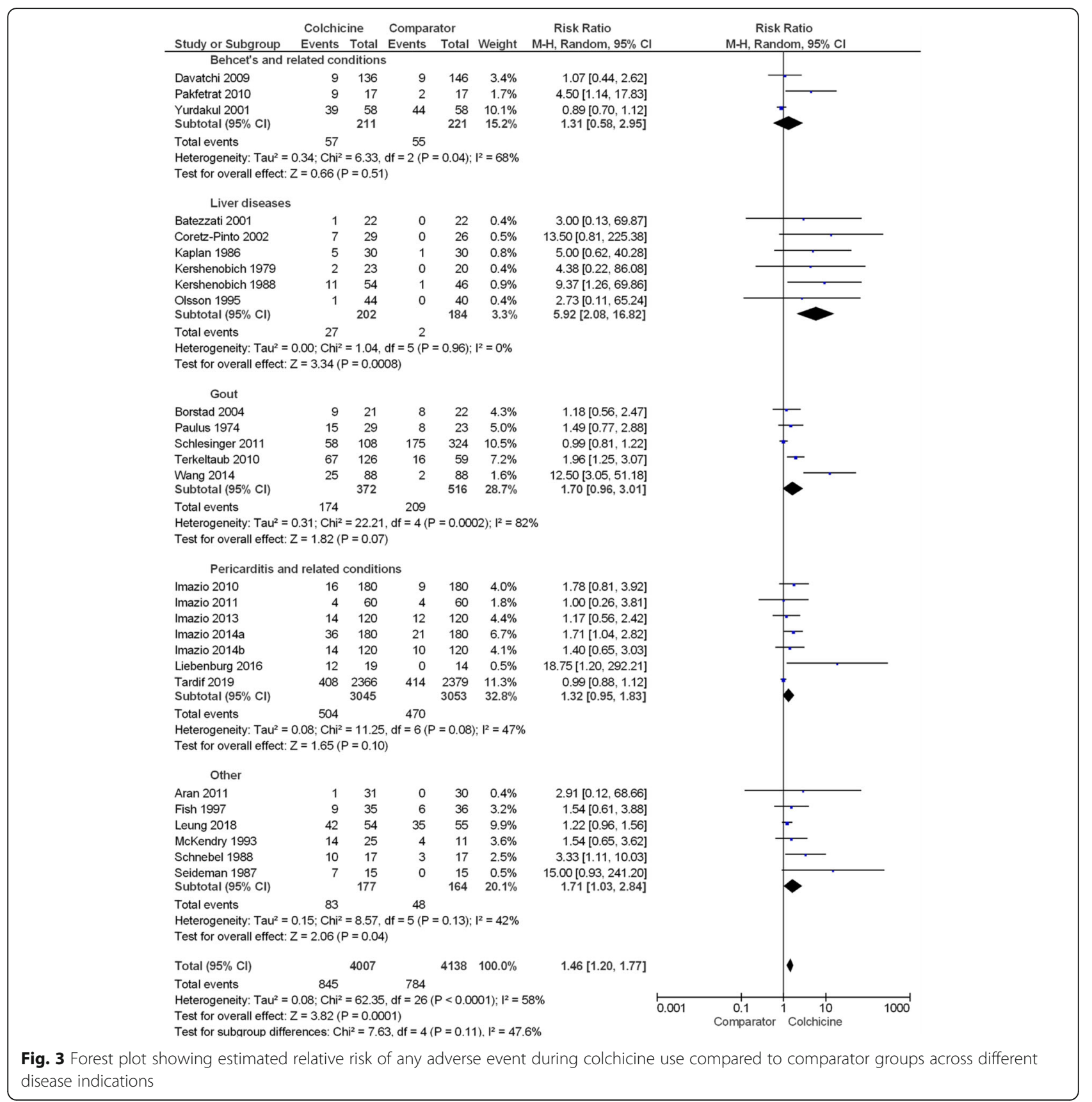

there was no significant difference in relative risk across different durations of drug exposure $(P=0.29)$ (Supplementary Figure 2), different colchicine daily dose categories $(P=0.70)$ (Supplementary Figure 3 ) or different colchicine cumulative dose categories $(P=$ 0.09) (Fig. 4).

The proportion of participants with any adverse event computed from all 35 studies in this review (in which prevalence was considered $0 \%$ if not reported) was $20.6 \%$ $(95 \%$ CI 19.5, 21.9) in colchicine users and 17.9\% $(95 \%$ CI $16.8,19.1)$ in comparator groups.

\section{Diarrhoea}

The number of participants with diarrhoea was reported by a total of 19 papers (Supplementary Table 2). From this data, $17.9 \%$ (95\% CI 16.8 19.1) of participants using colchicine reported diarrhoea compared to $13.1 \%$ (95\% CI 11.9, 14.3) of participants in comparator groups. The meta-analysis showed the overall estimated RR (95\% CI) of diarrhoea in colchicine users compared with pooled comparator groups was $2.44(1.62,3.69)(P<0.001)$ ((Supplementary Figure 4, Table 2). The difference in RR between placebo and active comparator groups was not 


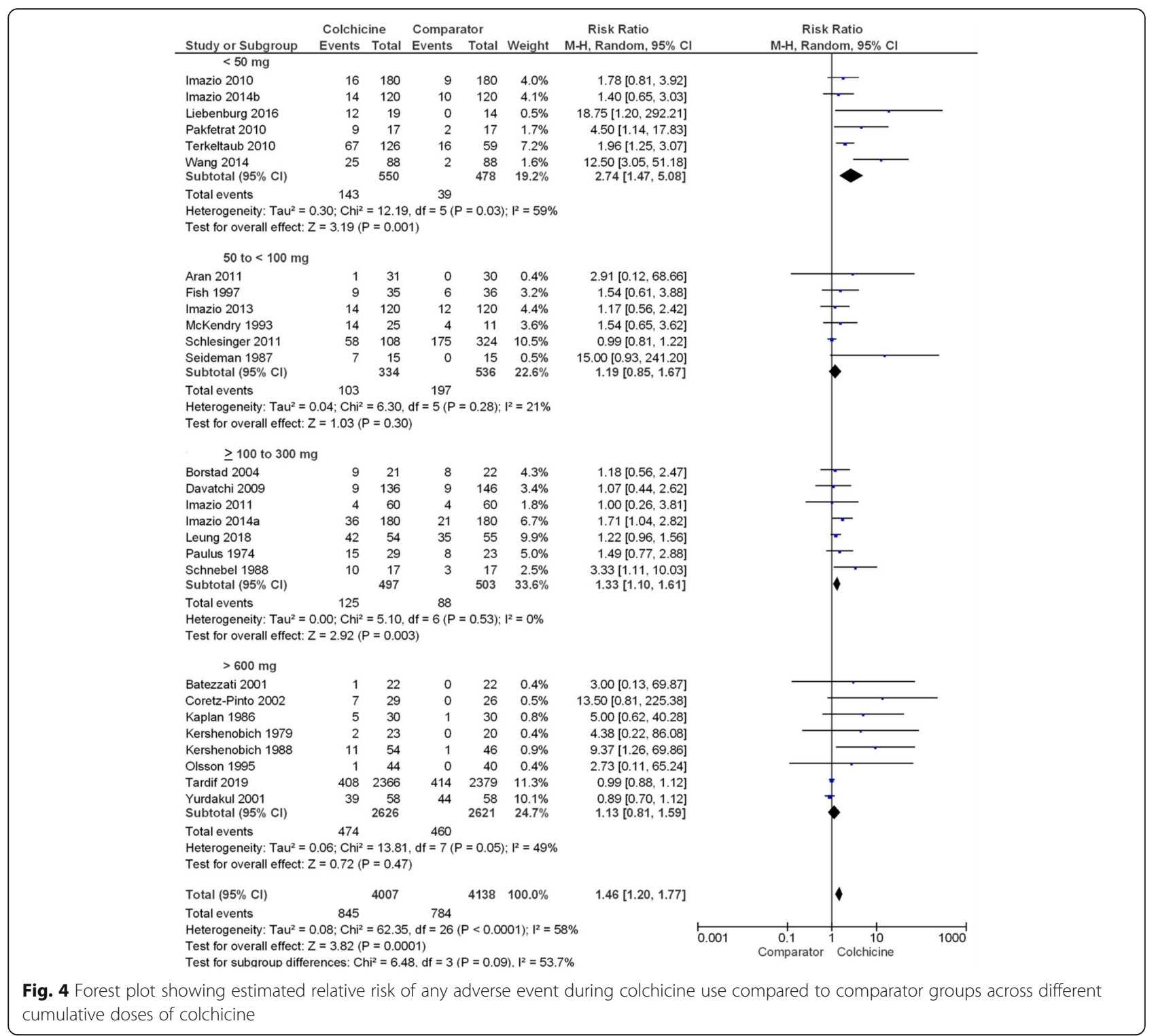

significant $(P=0.60)$. After exclusion of 6 studies involving participants with liver disease the RR $(95 \% \mathrm{CI})$ of diarrhoea in colchicine users vs comparator groups was similar at $2.14(1.40,3.26), P<0.001$ (Supplementary Table 3).

The proportion of participants with diarrhoea computed from all 35 studies in this review (in which prevalence was considered $0 \%$ if not reported) was $10.8 \%$ $(95 \%$ CI $9.9,11.7)$ in colchicine users and $6.1 \%$ (95\% CI $5.4,6.8$ ) in comparator groups.

\section{Gastrointestinal adverse event}

The number of participants with any gastrointestinal event was reported by 29 papers (Supplementary Table 2) and included diarrhoea, nausea, vomiting, abdominal pain, loss of appetite, bloating, constipation, melena and peptic ulcer
(Supplementary Table 4). From these 29 papers, 17.6\% (95\% CI 16.5, 18.8) of participants using colchicine reported a gastrointestinal event compared to $13.1 \%$ (95\% CI 12.1, 14.2) of participants in comparator groups. The overall RR $(95 \% \mathrm{CI})$ of gastrointestinal events in colchicine users compared with pooled comparator groups was 1.74 (1.32, 2.30), $P<0.001$ (Fig. 5, Table 2). The difference between placebo and active comparator groups was not significant $(P=0.32)$. After the exclusion of 6 studies involving participants with liver disease, the RR $(95 \% \mathrm{CI})$ of any gastrointestinal event in colchicine users vs comparator groups was similar at $1.60(1.22,2.10), P<0.001$ (Supplementary Table 3).

The proportion of participants with any gastrointestinal event computed from all 35 studies in this review (in which prevalence was considered $0 \%$ if not reported) 


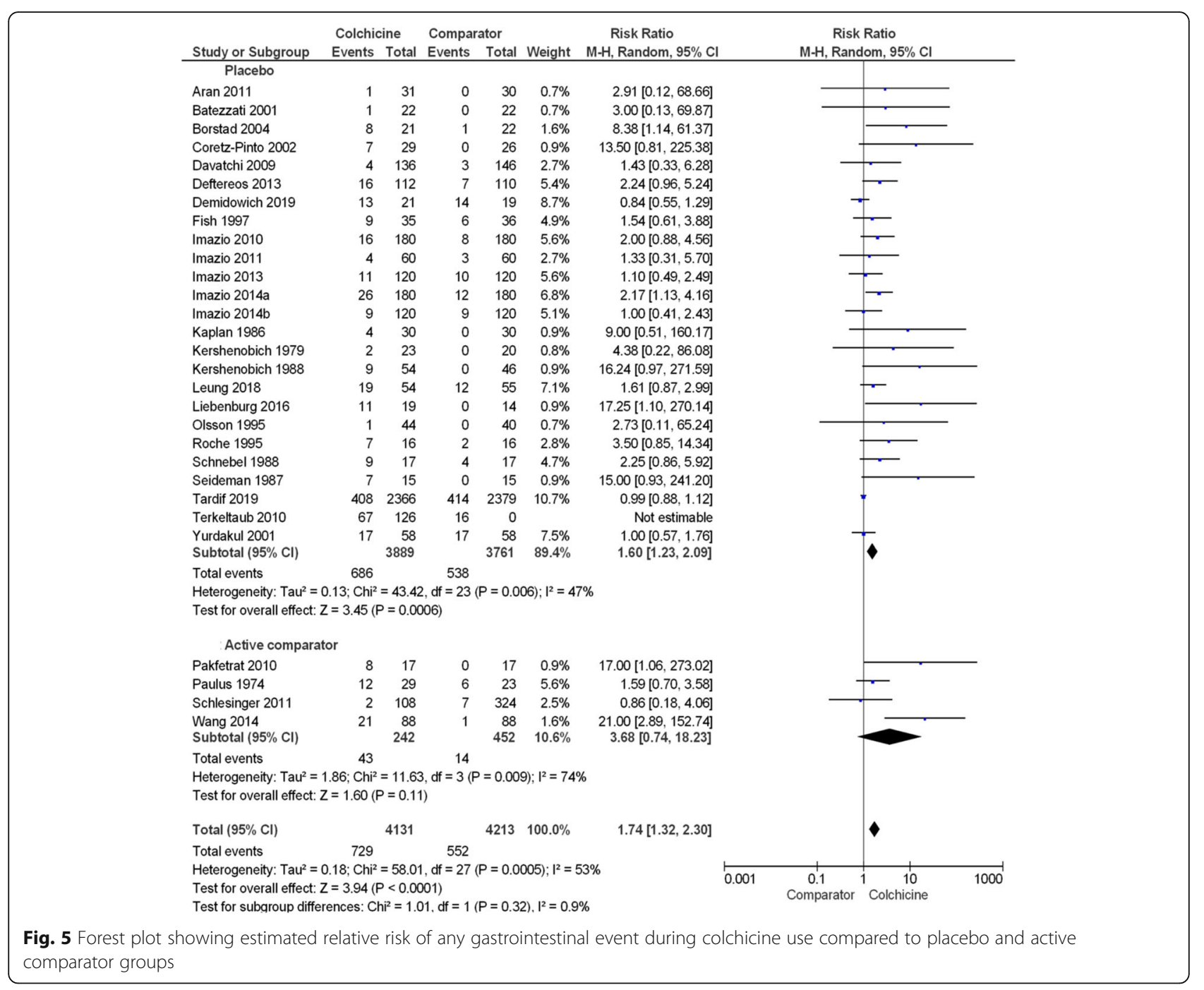

was $17.7 \%(95 \%$ CI $16.6,18.8)$ in colchicine users and $12.6 \%(95 \%$ CI $11.6,13.6)$ in comparator groups.

\section{Liver events}

The number of participants with liver events was reported by 13 papers (Supplementary Table 2) and included increased liver enzymes, hepatitis, hepatotoxicity and hepatic abnormalities (Supplementary Table 4). Pooled data from these papers showed 1.9\% (95\% CI 1.2, 2.8) of participants using colchicine reported a liver event compared to $1.1 \%$ (95\% CI $0.6,1.8)$ of participants in comparator groups. The overall RR (95\% CI) of liver events in colchicine users did not significantly differ from the pooled comparator groups: $1.61(0.86,3.02)$ (Supplementary Figure 5, Table 2). The difference between placebo and active comparator groups was also not significant. None of the included papers involved participants with liver diseases.
The proportion of participants with any liver event computed from all 32 studies in this review (in which prevalence was considered $0 \%$ if not reported) was $0.5 \%$ $(95 \%$ CI $0.3,0.7)$ in colchicine users and $0.3 \%$ (95\% CI $0.2,0.5)$ in comparator groups.

\section{Muscle events}

The number of participants with muscle events was reported by nine studies (Supplementary Table 2) and included myalgia, muscle cramps, elevated creatine phosphokinase and muscle weakness (Supplementary Table 5). Rhabdomyolysis was not mentioned in any study. All nine studies involved placebo comparator groups. Pooled data from these studies showed $4.2 \%$ (95\% CI 3.0, 5.7) of participants using colchicine reported a muscle event compared to 3.3\% (95\% CI 2.3, 4.7) of participants in placebo groups. The meta-analysis showed an overall non-significant RR $(95 \% \mathrm{CI})$ of muscle events in colchicine users of $1.25(0.80,1.93)$ (Fig. 6, 


\begin{tabular}{|c|c|c|c|c|c|c|c|c|c|c|}
\hline & Study or Subgroup & \multicolumn{2}{|c|}{ Colchicine } & \multicolumn{2}{|c|}{ Comparator } & Weight & $\begin{array}{c}\text { Risk Ratio } \\
\text { M-H, Fixed, } 95 \% \mathrm{Cl}\end{array}$ & \multicolumn{2}{|r|}{$\begin{array}{c}\text { Risk Ratio } \\
\text { M-H, Fixed, } 95 \% \mathrm{Cl}\end{array}$} & \\
\hline & Deftereos 2013 & 15 & 112 & 10 & 110 & $33.3 \%$ & $1.4 ?[0.69,3.14]$ & & + & \\
\hline & Demidowich 2019 & 4 & 21 & 6 & 19 & $20.8 \%$ & $0.60[0.20,1.82]$ & & $\Longrightarrow-$ & \\
\hline & Imazio 2010 & 0 & 180 & 1 & 180 & $4.9 \%$ & $0.33[0.01,8.13]$ & & & \\
\hline & Imazio 2011 & 0 & 60 & 0 & 60 & & Not estimable & & & \\
\hline & Imazio 2013 & 0 & 120 & 0 & 120 & & Not estimable & & & \\
\hline & Imazio 2014a & 0 & 180 & 0 & 180 & & Not estimable & & & \\
\hline & Imazio 2014b & 1 & 120 & 0 & 120 & $1.6 \%$ & $3.00[0.12,72.91]$ & & & \\
\hline & Leung 2018 & 12 & 54 & 8 & 55 & $26.1 \%$ & $1.53[0.68,3.44]$ & & $=-$ & \\
\hline & Total $(95 \% \mathrm{Cl})$ & & 872 & & 869 & $100.0 \%$ & $1.25[0.80,1.93]$ & & & \\
\hline & Total events & 37 & & 29 & & & & & & \\
\hline & $\begin{array}{l}\text { Heterogeneity. } \mathrm{Chi}^{2}= \\
\text { Test for overall effect: }\end{array}$ & $\begin{array}{l}3.04, d f= \\
Z=0.98(P\end{array}$ & $\begin{array}{l}5(P= \\
(P=0.3\end{array}$ & $\begin{array}{l}0.69) ; 1^{2}= \\
\text { 33) }\end{array}$ & $0 \%$ & & & 0.001 & $\begin{array}{ccc}0.1 & 1 & 10 \\
\text { Comparator } & \text { Colchicine }\end{array}$ & $\overrightarrow{1000}$ \\
\hline
\end{tabular}

Table 2). None of the studies involved participants with liver diseases.

The proportion of participants with muscle events computed from all 35 studies in this review (in which prevalence was considered $0 \%$ if not reported) was $0.8 \%$ $(95 \%$ CI $0.6,1.1)$ in colchicine users and $0.6 \%$ (95\% CI $0.4,0.9)$ in comparator groups.

\section{Haematology events}

The number of participants with haematology events was reported by eight studies (Supplementary Table 2) and included anaemia, bone marrow toxicity, leukopenia and purpura (Supplementary Table 4). All studies involved placebo comparator groups. Pooled data from these studies showed $0.6 \%(95 \%$ CI $0.3,0.9)$ of participants using colchicine reported a haematology event compared to $0.4 \%$ (95\% CI $0.2,0.7$ ) of participants in placebo groups. The occurrence of haematology events in colchicine or comparator groups was reported by three studies [21, 23, 37]. The meta-analysis showed an overall non-significant RR (95\% CI) of haematology events in $1.34(0.64,2.82)$ (Supplementary Figure 6, Table 2). None of the studies involved participants with liver diseases.

The proportion of participants with a haematology event computed from all 35 studies in this review (in which prevalence was considered $0 \%$ if not reported) was $0.4 \%(95 \%$ CI $0.2,0.6)$ in colchicine users and $0.3 \%$ (95\% CI 0.1, 0.4) in comparator groups.

\section{Sensory events}

No studies mentioned neuropathy-related adverse events. However, two studies involving placebo comparator groups reported other sensory events (Supplementary Table 2) which included dysthesia in the legs and paresthesia (Supplementary Table 5). From this data, the pooled prevalence of sensory events was $1.1 \%$ (95\% CI 0.2, 3.4) in colchicine users and 1.5\% (95\% CI $0.4,4.0)$ in placebo groups. The meta-analysis showed an overall non-significant RR $(95 \% \mathrm{CI})$ of sensory events in colchicine users of $1.35(0.27,6.74)$ (Supplementary
Figure 7, Table 2). None of the included papers involved participants with liver diseases.

The proportion of participants with any sensory events computed from all 35 studies in this review (in which prevalence was considered $0 \%$ if not reported) was $0.04 \%$ $(95 \%$ CI $0.0,0.1)$ in colchicine users and $0.07 \%$ (95\% CI $0.0,0.2)$ in comparator groups.

\section{Infectious events}

Seven studies reported various infectious events (Supplementary Table 2), including urinary tract infection, parotiditis, shingles, upper respiratory tract infection, nasopharyngitis and sinus congestion (Supplementary Table 5). From these papers, $0.4 \%$ (95\% CI 0.2, 0.6) of participants using colchicine reported an infectious event compared to $2.1 \%(95 \%$ CI $1.6,2.7)$ of participants in comparator groups. The overall RR (95\% CI) of infectious events in colchicine users compared with pooled comparator groups was non-significant: $1.03(0.70,1.51)$ (Supplementary Figure 8, Table 2). The difference between placebo and active comparator groups was not significant $(P=0.94)$. No study involved participants with liver diseases.

The proportion of participants with any infectious event computed from all 35 studies in this review (in which prevalence was considered $0 \%$ if not reported) was $2.4 \%(95 \%$ CI $2.0,2.9)$ in colchicine users and $2.8 \%$ (95\% CI 2.4, 3.4) in comparator groups.

\section{Death}

Death related to adverse events was specifically reported in three studies (Supplementary Table 2). No study reported deaths related to an adverse event.

\section{Miscellaneous events}

Miscellaneous adverse events reported by the included studies are summarised in Supplementary Table 6. These events were not meta-analysed but contributed to the 'any adverse event' category. 


\section{Discussion}

This systematic review and meta-analysis of randomised controlled trials indicate that overall, colchicine increases the rate of adverse events compared to both placebo and active comparators. Analysis of individual events demonstrated an increased risk for diarrhoea and gastrointestinal events in colchicine users, but no increase in the rate of other commonly cited adverse events, including liver, muscle, haematology, sensory or infectious events.

The mechanism by which colchicine induces diarrhoea and other gastrointestinal symptoms is not exactly known, but can be attributed to an increase in prostaglandin synthesis, intestinal secretion and gastrointestinal motility with this drug [41]. Although these symptoms can be clinical features of colchicine toxicity, they are usually mild, short-lived and reversible with dose reduction [12]. Serious adverse events associated with colchicine use, including neuropathy, myotoxicity and death were not reported in any trial included in the current analysis. These events may be more readily observed in less controlled environments evident in case reports involving colchicine over-dose, chronic renal diseases, interaction with concomitant medications and intravenous administration [42-49].

Analysis of adverse events in colchicine users showed no difference across different disease indications. Although overall, adverse events were numerically higher in patients with liver diseases, this risk was not significantly different from other disease indications. Furthermore, the sub-analysis excluding participants with liver disease showed similar adverse events rates to the main analysis. Although dose reduction is generally recommended when colchicine is used continuously in those with severe renal impairment, accurate conclusions regarding adverse events in this population could not be drawn from the current analysis.

There was notable heterogeneity across the clinical trials included in this review with regards to intervention methodology, including colchicine dose and treatment duration. However, sub-group analyses concluded that differences in drug use duration, daily dose or cumulative dose categories had no effect on the risk for adverse events. This contrasts with trials assessing the treatment of acute gout which report that high-dose colchicine results in a greater risk-to-benefit ratio. The paper reporting the AGREE trial included in the current analysis by Terkeltaub et al. [38], which directly compared two different doses of colchicine, found differences in adverse event rates between low and high dose groups, with $36 \%$ and $81 \%$ of participants having any adverse event, respectively. However, the short duration of treatment (1 to $6 \mathrm{~h}$ ) meant that the cumulative drug doses in both groups were low in the context of the other papers included in the meta-analysis, resulting in a nonsignificant effect of dose in the meta-analysis. The difference in adverse event rates between the two arms of this AGREE trial may relate to better surveillance of adverse events in this trial compared to previously reported trials, or the relatively high dose ( $4.8 \mathrm{mg}$ over $6 \mathrm{~h}$ ) of the high-dose colchicine group. This is the only published trial comparing two differing doses of colchicine so conclusions on the reason for this disparity are difficult to be definitive about.

The limitations of this study include the inability in assessing the occurrence of rarer adverse events when only short duration controlled clinical trials were included. Different methodology is required to assess the frequency of rarer adverse events. Furthermore, the aims of the majority of the included studies were not primarily to assess safety, resulting in limited availability of adverse event data for extraction. As only studies which specifically reported an adverse event as being present or absent were included in the meta-analyses, it is possible that the pooled results may have over-estimated the true occurrence of adverse events which were not reported in all papers. In addition, it is also possible that the pooled results may have under-estimated the true occurrence of adverse events which were not assessed (e.g. those requiring blood tests). There were few included participants with severely impaired renal function, so the ability to assess for safety in this group was limited. Clinical trials often recruit patients in a highly selective manner, including excluding those with co-morbidities, and therefore the results are not necessarily generalizable to a general patient population. In addition, the included studies spanned over 20 years and it is likely that participants in earlier studies are not representative of patients treated with colchicine in clinical practice today. Other limitations include the screening of titles, abstracts and full-texts being undertaken by a single reviewer, and the exclusion of non-English language publications.

The strengths of this study include the strict inclusion of only placebo or active comparator blinded trials which reduces the potential for bias; although the occurrence of diarrhoea in participants can lead to at the least the suspicion of being in the colchicine group. In addition, there was a wide range of included indications such as gout, familial Mediterranean fever, Behcet's disease and pericarditis, which leads to increased generalisability of the study results.

\section{Conclusions}

This meta-analysis provides reassurance that common adverse events with colchicine use are limited to diarrhoea and gastrointestinal events. Whilst these are not benign side effects in some individuals, they will settle on dose reduction or drug discontinuation. More serious 
adverse events during colchicine use, including liver and haematological changes, muscle toxicity, neuropathy and death are very infrequent in clinical trials.

\section{Supplementary information}

Supplementary information accompanies this paper at https://doi.org/10. 1186/s13075-020-2120-7.

Supplementary Table 1. Participant inclusion and exclusion criteria and adverse event assessment methods of included studies. Table 2. Frequency of any adverse event reported in colchicine and comparator groups. Table 3. Meta-analysis results showing pooled risk ratio of adverse events between colchicine and pooled comparator groups for studies not involving participants with liver diseases. Table 4. Number of participants in colchicine and comparator groups with adverse events related to gastrointestinal, liver and hematologic events. Table 5. Number of participants in colchicine and comparator groups with adverse events related to muscle, sensory, and infectious events. Table 6. Number of participants in colchicine and comparator groups with miscellaneous adverse events or death. Figure 1. Quality assessment results using the modified-Jadad score. Figure 2. Forest plot showing estimated relative risk of any adverse event during colchicine use compared to comparator groups across different durations of drug exposure. Figure 3. Forest plot showing estimated relative risk of any adverse event during colchicine use compared to comparator groups across different daily doses of colchicine. Figure 4. Forest plot showing estimated relative risk of diarrhoea during colchicine use compared to placebo and active comparator groups. Figure 5. Forest plot showing estimated relative risk of liver events during colchicine use compared to placebo and active comparator groups. Figure 6 . Forest plot showing estimated relative risk of hematology events during colchicine use compared to placebo (no active comparator studies). Figure 7. Forest plot showing estimated relative risk of sensory events during colchicine use compared to placebo (no active comparator studies). Figure 8 . Forest plot showing estimated relative risk of infectious events during colchicine use compared to placebo and active comparator groups.

\section{Abbreviations}

Cl: Confidence interval; PRISMA: Preferred Reporting Items for Systematic Reviews and Meta-Analyses; RR: Risk ratio

\section{Acknowledgements}

We would like to acknowledge Mr. Greg Gamble (Biostatistician, The University of Auckland) for his assistance with the meta-analyses.

\section{Authors' contributions}

PR and ND conceived the idea for the study. SS, KY, KA and ND extracted the data. SS and ND performed the statistical analysis. All authors wrote the manuscript and approved the final manuscript for publication.

\section{Authors' information}

NA

\section{Funding}

This study received no specific funding. Sarah Stewart is supported by an Auckland Medical Research Foundation Postdoctoral Fellowship.

\section{Availability of data and materials}

The data is available on request to the corresponding author.

\section{Ethics approval and consent to participate}

This is a systematic review and meta-analysis, ethical review is not required.

\section{Consent for publication}

Not relevant.

\section{Competing interests}

SS, KY, KA and PR declare no competing interests. Dr. Dalbeth reports grants and personal fees from AstraZeneca, grants from Amgen, personal fees from
Dyve, personal fees from Hengrui, personal fees from Horizon, personal fees from Kowa, personal fees from Abbvie, personal fees from Pfizer, personal fees from Janssen, outside the submitted work.

\section{Author details}

'Bone \& Joint Research Group, Faculty of Medicine, University of Auckland, Auckland, New Zealand. 'S School of Clinical Medicine, Faculty of Medicine, University of Queensland, Brisbane, Australia. ${ }^{3}$ Department of Rheumatology, Royal Brisbane Hospital, Bowen Bridge Road, Herston, QLD 4006, Australia.

Received: 2 August 2019 Accepted: 6 February 2020

Published online: 13 February 2020

\section{References}

1. Khanna D, et al. 2012 American College of Rheumatology guidelines for management of gout. Part 2: therapy and antiinflammatory prophylaxis of acute gouty arthritis. Arthritis Care Res (Hoboken). 2012;64(10):1447-61.

2. Terkeltaub RA, et al. Novel evidence-based colchicine dose-reduction algorithm to predict and prevent colchicine toxicity in the presence of cytochrome P450 3A4/P-glycoprotein inhibitors. Arthritis Rheum. 2011;63(8): 2226-37.

3. Evans $\mathrm{Tl}$, et al. A comprehensive investigation of inpatient intravenous colchicine use shows more education is needed. J Rheumatol. 1996;23(1): $143-8$.

4. Alabed S, et al. Colchicine for pericarditis. Cochrane Database Syst Rev. 2014;8:CD010652

5. Liberati Alessandro, Altman Douglas G, Tetzlaff Jennifer, Mulrow Cynthia, Gøtzsche Peter C, loannidis John PA, et al. The PRISMA statement for reporting systematic reviews and meta-analyses of studies that evaluate healthcare interventions: explanation and elaboration. BMJ. 2009;339:b2700.

6. Kaplan MM, et al. A prospective trial of colchicine for primary biliary cirrhosis. N Engl J Med. 1986;315(23):1448-54.

7. Battezzati PM, et al. Ten-year combination treatment with colchicine and ursodeoxycholic acid for primary biliary cirrhosis: a double-blind, placebocontrolled trial on symptomatic patients. Aliment Pharmacol Ther. 2001; 15(9):1427-34

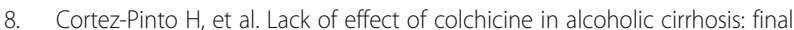
results of a double blind randomized trial. Eur J Gastroenterol Hepatol. 2002; 14(4):377-81.

9. Kershenobich D, et al. Treatment of cirrhosis with colchicine. A double-blind randomized trial. Gastroenterology. 1979;77(3):532-6.

10. Kershenobich $\mathrm{D}$, et al. Colchicine in the treatment of cirrhosis of the liver. $\mathrm{N}$ Engl J Med. 1988;318(26):1709-13.

11. Olsson $\mathrm{R}$, et al. Colchicine treatment of primary sclerosing cholangitis. Gastroenterology. 1995;108(4):1199-203.

12. Dinarello CA, et al. Colchicine therapy for familial mediterranean fever. A double-blind trial. N Engl J Med. 1974;291(18):934-7.

13. McKendry RJ, et al. Therapeutic value of colchicine in the treatment of patients with psoriatic arthritis. Ann Rheum Dis. 1993;52(11):826-8.

14. Roche $\mathrm{N}$, et al. Nasal response to capsaicin in patients with allergic rhinitis and in healthy volunteers: effect of colchicine. Am J Respir Crit Care Med. 1995;151(4):1151-8.

15. Schlesinger $\mathrm{N}$, et al. Canakinumab reduces the risk of acute gouty arthritis flares during initiation of allopurinol treatment: results of a double-blind, randomised study. Ann Rheum Dis. 2011;70(7):1264-71.

16. Aran S, Malekzadeh S, Seifirad S. A double-blind randomized controlled trial appraising the symptom-modifying effects of colchicine on osteoarthritis of the knee. Clin Exp Rheumatol. 2011;29(3):513-8.

17. Bessissow A, et al. Colchicine for prevention of perioperative atrial fibrillation in patients undergoing lung resection surgery: a pilot randomized controlled study. Eur J Cardiothorac Surg. 2018;53(5):945-51.

18. Borstad GC, et al. Colchicine for prophylaxis of acute flares when initiating allopurinol for chronic gouty arthritis. J Rheumatol. 2004;31(12):2429-32.

19. Cohen $A B$, et al. $A$ controlled trial of colchicine to reduce the elastase load in the lungs of ex-cigarette smokers with chronic obstructive pulmonary disease. Am Rev Respir Dis. 1991;143(5 Pt 1):1038-43.

20. Das SK, et al. A randomized controlled trial to evaluate the slow-acting symptom modifying effects of a regimen containing colchicine in a subset of patients with osteoarthritis of the knee. Osteoarthr Cartil. 2002;10(4):247-52. 
21. Davatchi F, et al. Colchicine versus placebo in Behcet's disease: randomized, double-blind, controlled crossover trial. Mod Rheumatol. 2009;19(5):542-9.

22. Deftereos S, et al. Colchicine treatment for the prevention of bare-metal stent restenosis in diabetic patients. J Am Coll Cardiol. 2013;61(16):1679-85.

23. Demidowich AP, et al. Effects of colchicine in adults with metabolic syndrome: a pilot randomized controlled trial. Diabetes Obes Metab. 2019; 21(7):1642-51.

24. Fish JE, et al. An evaluation of colchicine as an alternative to inhaled corticosteriods in moderate asthma. National Heart, Lung, and Blood Institute's asthma clinical research network. Am J Respir Crit Care Med. 1997; 156(4 Pt 1):1165-71.

25. Imazio $\mathrm{M}$, et al. COlchicine for the prevention of the post-pericardiotomy syndrome (COPPS): a multicentre, randomized, double-blind, placebocontrolled trial. Eur Heart J. 2010;31(22):2749-54.

26. Imazio $\mathrm{M}$, et al. Colchicine for recurrent pericarditis (CORP): a randomized trial. Ann Intern Med. 2011;155(7):409-14.

27. Imazio $\mathrm{M}$, et al. A randomized trial of colchicine for acute pericarditis. $\mathrm{N}$ Engl J Med. 2013;369(16):1522-8.

28. Imazio $\mathrm{M}$, et al. Colchicine for prevention of postpericardiotomy syndrome and postoperative atrial fibrillation: the COPPS-2 randomized clinical trial. JAMA. 2014:312(10):1016-23.

29. Imazio M, et al. Efficacy and safety of colchicine for treatment of multiple recurrences of pericarditis (CORP-2): a multicentre, double-blind, placebocontrolled, randomised trial. Lancet. 2014;383(9936):2232-7.

30. Leung $Y Y$, et al. Colchicine lack of effectiveness in symptom and inflammation modification in knee osteoarthritis (COLKOA): a randomized controlled trial. Osteoarthr Cartil. 2018;26(5):631-40.

31. Liebenberg JJ, Dold CJ, Olivier LR. A prospective investigation into the effect of colchicine on tuberculous pericarditis. Cardiovasc J Afr. 2016;27(6): 350-5.

32. Masuda K, et al. Double-masked trial of cyclosporin versus colchicine and long-term open study of cyclosporin in Behcet's disease. Lancet. 1989; 1(8647):1093-6.

33. Pakfetrat $\mathrm{A}$, et al. Comparison of colchicine versus prednisolone in recurrent aphthous stomatitis: a double-blind randomized clinical trial. Clin Invest Med. 2010;33(3):E189-95.

34. Paulus HE, et al. Prophylactic colchicine therapy of intercritical gout. A placebo-controlled study of probenecid-treated patients. Arthritis Rheum. 1974;17(5):609-14.

35. Schnebel BE, Simmons JW. The use of oral colchicine for low-back pain. A double-blind study. Spine (Phila Pa 1976). 1988;13(3):354-7.

36. Seideman P, Fjellner B, Johannesson A. Psoriatic arthritis treated with oral colchicine. J Rheumatol. 1987;14(4):777-9.

37. Tardif J-C, et al. Efficacy and safety of low-dose colchicine after myocardial infarction. N Engl J Med. 2019;381:2497-05.

38. Terkeltaub RA, et al. High versus low dosing of oral colchicine for early acute gout flare: twenty-four-hour outcome of the first multicenter, randomized, double-blind, placebo-controlled, parallel-group, dosecomparison colchicine study. Arthritis Rheum. 2010;62(4):1060-8.

39. Wang $Y$, et al. Chuanhu anti-gout mixture versus colchicine for acute gouty arthritis: a randomized, double-blind, double-dummy, non-inferiority trial. Int J Med Sci. 2014;11(9):880-5.

40. Yurdakul S, et al. A double-blind trial of colchicine in Behcet's syndrome Arthritis Rheum. 2001;44(11):2686-92.

41. Levy M, Spino M, Read SE. Colchicine: a state-of-the-art review. Pharmacotherapy. 1991;11(3):196-211.

42. Alayli G, et al. Acute myopathy in a patient with concomitant use of pravastatin and colchicine. Ann Pharmacother. 2005;39(7-8):1358-61.

43. Chattopadhyay I, et al. Colchicine induced rhabdomyolysis. Postgrad Med J. 2001;77(905):191-2.

44. Ducloux D, et al. Colchicine myopathy in renal transplant recipients on cyclosporin. Nephrol Dial Transplant. 1997;12(11):2389-92.

45. Eleftheriou $\mathrm{G}$, et al. Colchicine-induced toxicity in a heart transplant patient with chronic renal failure. Clin Toxicol (Phila). 2008:46(9):827-30.

46. Lee $K Y$, et al. Two cases of acute leukopenia induced by colchicine with concurrent immunosuppressants use in Behcet's disease. Yonsei Med J. 2008;49(1):171-3.

47. Montseny JJ, Meyrier A, Gherardi RK. Colchicine toxicity in patients with chronic renal failure. Nephrol Dial Transplant. 1996;11(10):2055-8.

48. Wilbur K, Makowsky M. Colchicine myotoxicity: case reports and literature review. Pharmacotherapy. 2004;24(12):1784-92.
49. Yoon $\mathrm{KH}$. Colchicine induced toxicity and pancytopenia at usual doses and treatment with granulocyte colony-stimulating factor. J Rheumatol. 2001; 28(5):1199-200.

\section{Publisher's Note}

Springer Nature remains neutral with regard to jurisdictional claims in published maps and institutional affiliations.
Ready to submit your research? Choose BMC and benefit from:

- fast, convenient online submission

- thorough peer review by experienced researchers in your field

- rapid publication on acceptance

- support for research data, including large and complex data types

- gold Open Access which fosters wider collaboration and increased citations

- maximum visibility for your research: over $100 \mathrm{M}$ website views per year

At $\mathrm{BMC}$, research is always in progress.

Learn more biomedcentral.com/submissions 